\title{
Classical groups over division rings of characteristic two
}

\section{William M. Pender}

\begin{abstract}
The notion of quadratic form over a field of characteristic two is extended to an arbitrary division ring of characteristic two with an involution of the first kind. The resulting isometry groups are shown to have a simple normal subgroup and the structure of the factor group is calculated. It is indicated how one may define and analyse all the classical groups in a unified manner by means of quadratic forms.
\end{abstract}

\section{Introduction}

Suppose $k$ is a division ring, commutative or not, of characteristic two with an involution $J$ of the first kind (that is, $J$ is an involutory or trivial antiautomorphism of $k$ fixing every element of the centre $z$ of $k$ ). It is well-known that the definition of the classical groups over $k$ is more complicated than over other types of division rings. Firstly in division rings of characteristic not two or with involutions of the second kind, every symmetric element is a trace (a trace is an element of the form $\lambda+\lambda^{J}$ ), but in $k$ there may be symmetric elements which are not traces this is certainly the case when $k$ has finite dimension over $z$. The classical groups over $k$ are always defined in terms of trace-valued hermitian $J$-sesquilinear forms (hermitian forms $f$ such that $f(x, x)$ is a trace for all $x$ in the space) because the isometry groups of non-trace-valued hermitian forms can always be described in terms of trace-valued forms (Dieudonné [5]).

Received 26 April 1972. Communicated by G.E. Wall. 191 
Furthermore if $k$ is a field then symmetric bilinear forms will give only the symplectic group, and to define the orthogonal group one must use quadratic forms, each of which is associated with a trace-valued symmetric bilinear form but not determined by it. This quadratic form may be defective, that is it may be non-degenerate but have a degenerate symmetric bilinear form associated with it. The symplectic groups over $k$ may be regarded as defective orthogonal groups where the defect is as large as possible (Dieudonné [5]).

The purpose of this paper is to investigate new classical groups which arise in the case when $k$ is not commutative as the isometry groups of $J$-quadratic forms over $k$. A $J$-quadratic form is a map from a vector space over $k$ to the factor group of $k$ modulo the $z$-space of traces of $k$, and has associated with it a trace-valued hermitian $J$-sesquilinear form. As is the case for fields, these forms may be defective and the usual unitary groups over $k$ (defined in terms of trace-valued hermitian $J$-sesquilinear forms alone) may be regarded as isometry groups of $J$-quadratic forms whose defect is as large as possible.

All the classical groups may be defined in the same way as isometry groups of $J$-quadratic forms if this notion is extended to involutions of the second kind and division rings of characteristic not two using skew-traces (elements of the form $\lambda-\lambda^{J}$ ) as well as traces. The development of the structure theorems given here over $k$ does not use arguments essentially depending on characteristic two, and can easily be extended to give a unified account of all the indefinite classical groups. When every symmetric element is a trace, however, the quadratic form is completely determined by the associated sesquilinear form, in fact for symplectic groups over fields of characteristic not two the quadratic form is trivial and the definitions must be reworded in terms of both forms.

The idea of a $J$-quadratic form was first developed over a quaternionic division ring (in which the trace space may be taken as the centre) by Seip-Hornix [8], so that her treatment of Clifford algebras over quaternionic division rings would not be restricted by characteristic. The possibility of generalizing Seip-Hornix's definition is mentioned by Tits in [9] but without explanation. The algebraic groups he there denotes by 
${ }^{1} D_{n, r}(d)$ and ${ }^{2} D_{n, r}(d)$ are said to correspond to isometry groups of nondefective $J$-quadratic forms of Witt index $r$ over a division ring of finite dimension $d^{2}$ over the centre.

We shall prove the fundamental structure theorems in the case when the Witt index is non-zero and $k$ is commutative or non-commutative. That is we shall prove that a certain normal subgroup of the isometry group is simple, and produce a norm on the isometry group which determines the structure of the resulting factor group. The proof of simplicity follows the method of I wasawa [7] and the construction of the "Wall norm" is a simple extension of Wall's paper [10] using the same arguments. The arguments concerning $G F(2)$ are often omitted, and can usually be found in Dieudonné [5].

\section{Preliminaries on division rings}

With the division ring $k$ as before, it is clear that both the set $s$ of symmetric elements and the set $\boldsymbol{l}$ of trace elements form vector spaces over $z$. Also the map $\operatorname{Tr}: k \rightarrow k$ mapping each $\lambda \in k$ to $\lambda+\lambda^{J}$ is $z$-linear with kernel $s$ and image $l$, hence the $z$-codimension of $s$ equals the $z$-dimension of $l$. When $k$ has finite dimension $m^{2}$ over $\boldsymbol{z}$, then $s$ has dimension $\frac{1}{2}(m+1)$ and so $l$ has dimension $\frac{1}{2} m(m-1)$. When $k$ has infinite dimension over $z$ it is easily shown that $l$ also has infinite dimension.

The trace space is closed under inverses, since if $\alpha=\lambda+\lambda^{J} \epsilon 2^{\#}$ then $\alpha^{-1}$ is symmetric and so $\alpha^{-1} \lambda\left(\alpha^{-1}\right)^{J}+\left(\alpha^{-1} \lambda\left(\alpha^{-1}\right)^{J}\right)^{J}=\alpha^{-1} \in 2$. When 1 is a trace similar arguments show that every norm $\lambda \lambda^{J}$ is a trace and that $l$ is closed under the operation of squaring. Also when $l$ is a trace, either $k$ is generated by its traces or the trace space coincides with the centre (the analagous theorem for symmetric elements is proven in Dieudonné [2]). For then the division subring $k^{\prime}$ generated by $l$ is invariant under all inner automorphisms of $k$, since if $\alpha \in l$ and $\mu \in k^{\#}$ then $\mu^{-1} \alpha \mu=\left(\mu^{-1} \alpha\left(\mu^{-1}\right)^{J}\right)\left(\mu^{J} \mu\right) \in k^{\prime}$. Now by the Cartan-BrauerHua Theorem (Brauer [1]), $k^{\prime}$ is either $z$ or $k$. 


\section{Quadratic forms}

Suppose $k$ is a commutative or non-commutative division ring of characteristic two with an involution $J$ of the first kind. Let $z$ be the centre of $k$ and let $s$ and $l$ be the vector spaces over $z$ formed respectively by the symmetric elements of $k$ and the trace elements of $k$. Suppose $E$ is a left vector space of finite or infinite dimension $n$ over $k$.

LEMMA 1.1. (a) For each $\lambda \in k^{\#}$, the mop $k \rightarrow k$ defined by

$$
\alpha \rightarrow \lambda \alpha \lambda^{J} \text { for all } \alpha \in k
$$

is a z-linear automorphism of the z-space $k$ leaving invariant the trace space 2 . Hence the map induces a z-linear automorphism of the factor space $k / 2$, and we shall denote the image of $\alpha+2$ in $k / 2$ by $\lambda(\alpha+2) \lambda^{J}$.

(b) The map $\operatorname{Tr}: k \rightarrow Z$ defined by

$$
\alpha \rightarrow \alpha+\alpha^{J} \text { for all } \alpha \in k
$$

is a z-linear epimorphism from $k$ to $l$ with kernel $s$. Hence the map induces a z-linear epimorphism $\operatorname{Tr}: k / l+l$, and we shall denote the image of $\alpha+\tau$ in $k / 2$ by $\operatorname{Tr}(\alpha+z)$.

(c) The antiautomorphism $J$ of $k$ regarded as a z-linear automorphism of the z-space $k$ induces the identity map on the factor space $k / 2$.

DEFINITION 1.2. A J-quadratic form on $E$ is a map $q: E+k / 2$ satis fying:

(i) for all $x \in E$ and all $\lambda \in k$,

$$
q(\lambda x)=\lambda q(x) \lambda^{J} ;
$$

(ii) there exists a $J$-sesquilinear form $f$ on $E$ such that for all $x, y \in E$,

$$
f(x, y) \in q(x+y)+q(x)+q(y) .
$$

The form $f$ is unique, since if $g$ is another such form then $f+g$ has all its values in $l$, but $l$ is strictly less than $k$ and so $f+g$ 
is the zero form. We call this unique form $f$ the polar form of $q$.

LEMMA 1.3. Suppose $q$ is a J-quadratic form on $E$. Then the polar form $f$ of $q$ is trace-valued hermitian, and for all $x \in E$,

$$
f(x, x)=\operatorname{Tr}(q(x)) .
$$

Proof. When $k$ is a field, $f$ is alternating and the result is trivial, so assume $k$ is not a field. Suppose $x \in E$ and $\lambda, \mu \in k$, and put $q(x)=\alpha+2$. Then

$$
\operatorname{Tr}(f(\lambda x, \mu x))=\operatorname{Tr}\left((\lambda+\mu) \alpha(\lambda+\mu)^{J}+\lambda \alpha \lambda^{J}+\mu \alpha \mu^{J}\right),
$$

and so

$$
\operatorname{Tr}\left(\lambda f(x, x) \mu^{J}\right)=\lambda\left(\alpha+\alpha^{J}\right) \mu^{J}+\mu\left(\alpha+\alpha^{J}\right) \lambda^{J}
$$

Hence $\lambda(f(x, x)+\operatorname{Tr}(q(x))) \mu^{J}$ is symetric for all $\lambda, \mu \in k$, and so $f(x, x)=\operatorname{Tr}(q(x))$ since $s$, since $s$ is strictly less than $k$.

Suppose $x, y \in E$. Then from the first paragraph

$$
f(x+y, x+y)=\operatorname{Tr}(q(x+y)),
$$

and so

$$
\begin{aligned}
\operatorname{Tr}(q(x))+\operatorname{Tr}(q(y))+f(x, y)+f(y, x) & \\
& =\operatorname{Tr}(q(x))+\operatorname{Tr}(q(y))+\operatorname{Tr}(f(x, y)) .
\end{aligned}
$$

Hence $f(y, x)=f(x, y)^{J}$ and $f$ is hermitian.

1.4. Degeneracy and defect. One can easily verify that the abelian group $s / l$ can be regarded as a left vector space over $k$ with the action of $\lambda \in k$ on $\alpha+z \in s / z$ defined to be $\lambda(\alpha+z) \lambda^{J}$. Suppose that $q$ is a $J$-quadratic form on $E$ with polar form $f$. We define the radical $R(E, q)$ of $(E, q)$ to be the radical of the polar form $f$. Then the $q$-image of $R(E)$ is contained in $s / Z$, and in fact the restriction

$$
\left.q\right|_{R(E)}: R(E) \rightarrow s / 2
$$

is $k$-linear when $s / 2$ is regarded as a $k$-space in the above fashion. The rank of this map is called the defect of $q$, and the image is called the defect space of $q$. The nullity of the map is called the degeneracy of $q$, and the kernel is called the kernel of $q, K(E, q)$. 
The forms $f$ and $q$ induce forms $\bar{f}$ and $\bar{q}$ on the factor space $\bar{E}$ of $E$ by $K(E)$ in the canonical way, and clearly $\bar{f}$ is the polar form of $\bar{q}$. We call $(\bar{E}, \bar{q})$ the corresponding non-degenerate space and form.

LEMMA 1.5. (a) Suppose $f$ is a trace-valued J-hermitian form on $E$. Suppose $\left\{e_{i} \mid i \in I\right\}$ is a basis of $E$ and suppose $p:\left\{e_{i} \mid i \in I\right\} \rightarrow k / 2$ is a map satisfying

$$
\operatorname{Tr}\left(p\left(e_{i}\right)\right)=f\left(e_{i}, e_{i}\right) \text { for alz } i \in I \text {. }
$$

Then there is a unique $J$-quadratic form on $E$ extending $p$ with polar form $f$, and if $f$ is non-degenerate then this form is non-defective.

(b) AZso if $s / 2$ is regarded as a $k$-space in the sense of 1.4 , then there is a unique J-quadratic form on $E \oplus(\varepsilon / Z)$ with polar form the trivial extension of $f$ whose restriction to $E$ extends $p$ and whose restriction to $s / Z$ is the identity, and the defect space of this form is the whole of $s / 2$.

Proof. We prove the first part, then the second part follows easily. Define the map $q: E \rightarrow k / 2$ as follows: for each element $\sum_{i \in F} \lambda_{i} e_{i} \in E$, where $F$ is a finite subset of $I$ ordered arbitrarily by <, define

$$
q\left(\sum_{i \in F} \lambda_{i} e_{i}\right)=\sum_{i \in F} \lambda_{i} p\left(e_{i}\right) \lambda_{i}^{J}+\left(\sum_{i<j} \lambda_{i} f\left(e_{i}, e_{j}\right) \lambda_{j}^{J}+2\right) \text {, }
$$

then it is straightforward to verify that $q$ is a $J$-quadratic form on $E$ with polar form $f$. Furthermore any such $J$-quadratic form must satisfy the above equation, and so $q$ is unique.

1.6 Singular points and spaces. Suppose $q$ is a $J$-quadratic form on $E$ with polar form $f$. A vector $x \in E$ is called singular if $q(x)=0$, and a subspace $V$ of $E$ is called singular if every vector in $V$ is singular. The Witt index of $q$ is defined to be the maximum dimension of a singular subspace of $(E, q)$ disjoint from $R(E)$, that is the maximum dimension of a singular subspace of $(\bar{E}, \bar{q})$. We call $q$ definite if it has Witt index zero, and indefinite otherwise.

A subspace $V$ of $E$ is singular if and only if 
(a) $V$ is isotropic, and

(b) $V$ has a basis of singular vectors.

In particular, the Witt index of $q$ is at most half the rank of $f$.

LEMMA 1.7. (a) Suppose $q$ is a J-quadratic form on $E$ with polar form $f$. Suppose $\left(e_{1}, \ldots, e_{r}\right)$ is a basis of a finite dimensional singular subspace of $(E, q)$ disjoint from $R(E)$. Then we can choose $d_{1}, \ldots, d_{r} \in E$ such that

(i) $\left\langle d_{1}, \ldots, d_{p}\right\rangle$ is singuzar;

(ii) $f\left(e_{i}, a_{j}\right)=\delta_{i j}$.

(b) If $q$ is indefinite and $f$ is not the zero form, then $(E, q)$ has a basis of singular vectors outside $R(E)$.

Proof. (a) Since $f$ is trace-valued we can choose $d_{1}^{\prime}, \ldots, d_{r}^{\prime} \in E$ such that $\left\langle d_{l}^{\prime}, \ldots, d_{r}^{\prime}\right\rangle$ is isotropic and $f\left(e_{i}, d_{j}^{\prime}\right)=\delta_{i j}$. For each. $i=1, \ldots, r$ define $d_{i}=d_{i}^{\prime}+\alpha_{i} e_{i}$ where $\alpha_{i} \in q\left(d_{i}^{\prime}\right)$.

(b) Choose e $\in E \backslash R(E)$ singular. Choose a basis $\left\{x_{i} \mid i \in I\right\}$ of $e^{\perp}$ with $x_{1}=e$, and choose $y \in E \backslash e^{l}$. Then $\left\{y+x_{i} \mid i \in I\right\} \cup\{e\}$ spans $E$ and is linearly independent, so is a basis of $E$. For each $i \in I$ the plane $\left\langle e, y+x_{i}\right\rangle$ is an $f$-non-degenerate subspace of $(E, q)$ and so by $(a)$ contains a singular vector $e_{i}$ such that $f\left(e, e_{i}\right)=1$. Now $\left\{e_{i} \mid i \in I\right\} \cup\{e\}$ is a basis of $(E, q)$ outside $R(E)$.

1.8 Semi-singular points and spaces. Suppose $q$ is a $J$-quadratic form on $E$ with polar form $f$. A vector $x \in E$ is called semi-singular if $q(x) \in q(R)$, and a subspace $V$ of $E$ is called semi-singular if every vector in $V$ is semi-singular and $V$ is isotropic.

Thus a subspace $V$ of $E$ is semi-singular if and only if

(a) $V$ is isotropic, and

(b) $V$ has a basis of semi-singular vectors. 
If $q$ is non-defective, then semi-singular and singular coincide. If the defect space of $q$ is the whole of $s$, then semi-singular and isotropic coincide. If $J \neq 1$, or if $k$ is a field and $q(R)<k$, then a subspace $V$ is semi-singular if and only if every vector of $V$ is semi-singular (for if every vector of $V$ is semi-singular, then all the values of $\left.f\right|_{V}$ lie in the defect space of $q$ which here is strictly less than $k$, and so $V$ is isotropic).

Suppose $V$ is a semi-singular subspace of $(E, q)$ disjoint from $R(E)$. Choose a basis $\left\{e_{i} \mid i \in I\right\}$ of $V$, and for each $i \in I$ choose $t_{i} \in R(E)$ so that $q\left(t_{i}\right)=q\left(e_{i}\right)$. Then the subspace $\left(e_{i}+t_{i} \mid i \in I\right)$ is singular of the same dimension as $V$. We conclude that the Witt index of $q$ equals the maximum dimension of a semi-singular subspace of $(E, q)$ disjoint from $R(E)$.

1.9 Hyperbolic planes. Suppose $q$ is a $J$-quadratic form on $E$ with polar form $f$. A hyperbolic pair in $(E, q)$ is a pair $(e, d)$ of singular vectors such that $f(e, d)=1$. The subspace spanned by a hyperbolic pair is called a hyperbolic plane of $(E, q)$. By Lemma 1.6 every $f$-non-degenerate subplane of $(E, q)$ containing non-zero singular vectors is a hyperbolic plane.

LEMMA 1.10. (a) Suppose $q$ is a J-quadratic form on $E$ with polar form $f$. If $k$ is a field then every hyperbolic plane of $(E, q)$ contains exactly two singular lines. If $k$ is not a field then every hyperbolic plane of $(E, q)$ contains infinitely many singular lines.

(b) If $q$ is indefinite and $n \geq 3$ and $k$ is not $G F(2)$, then every line of $E$ outside $R(E)$ is the intersection of two hyperbolic pranes.

Proof. If $(e, d)$ is a hyperbolic pair then the line $(e+\lambda d)$ is singular if and only if $\lambda \in Z$. Part $(a)$ now follows easily. The proof of (b) is well-known when $k$ is a field (Dieudonne [5]) so we assume $k$ is not a field.

Suppose $x \in E \backslash R(E)$ is singular. Choose $y \in E$ singular so $(x, y)$ is a hyperbolic pair. Choose $z \in\langle x, y\rangle^{\perp}, z \neq 0$. Then $\langle x, y\rangle$ and $(x, y+z)$ are distinct hyperbolic planes containing $\langle x\rangle$. 
Suppose $x \in E \backslash R(E)$ is non-singular. Since $E$ has a basis of singular vectors we can choose $y \in E$ singular so $f(x, y)=1$. Put $z=x+f(x, x) y$, then $z \in x^{+}$and $z k y^{\perp}$. Choose $t \in(x, y)^{\perp}$ non-zero, then $z+t \in x^{\perp}$ and $z+t k y^{1}$. By part (a) and the assumption that $k$ is not a field, the hyperbolic plane $(z+t, y)$ contains a singular line $\langle e\rangle$ distinct from both $\langle y\rangle$ and $\langle z+t\rangle$, and hence not orthogonal to $\langle x\rangle$ nor in $\langle x, y\rangle$. Now $\langle x, y\rangle$ and $(x, e)$ are distinct hyperbolic planes containing $(x)$.

\section{Unitary groups}

Suppose that the division ring $k$ and the vector space $E$ are as in the first section. Suppose that $q$ is a $J$-quadratic form on $E$ of Witt index $v$ with polar form $f$ and defect space $m$.

2.1 Images and multiples of quadratic forms. Suppose $k^{\prime}$ is a division ring isomorphic to $k$ and $E^{\prime}$ is a left vector space over $k^{\prime}$. Suppose $\sigma: k^{\prime} \rightarrow k$ is an isomorphism and $X: E^{\prime} \rightarrow E$ is a $\sigma$-linear map. Define the involution $J^{\prime}$ of $k^{\prime}$ to be $\sigma J \sigma^{-1}$; then $J^{\prime}$ is of the first kind and the trace space $l^{\prime}$ of $k^{\prime}$ is $l^{\sigma^{-1}}$. Define the map $q^{X}: E^{\prime} \rightarrow k^{\prime} / 2^{\prime}$ by

$$
q^{X}(x)=q(x X)^{\sigma^{-1}}
$$

then $q^{X}$ is a $J^{\prime}$-quadratic form on $E^{\prime}$ with polar form the $J^{\prime}$-hermitian form $f^{X}$ on $E^{\prime}$ defined by $f^{X}(x, y)=f(x X, y X)^{\sigma^{-1}}$.

Suppose $\rho \in k^{\#}$. Then right multiplication of $k$ by $\rho$ is a z-linear automorphism of $k$ mapping $l$ to $\tau_{\rho}$, and $K=\rho^{-1} J \rho$ is an antiautomorphism of $k$. Define the map $q \rho: E \rightarrow k / l_{\rho}$ by

$$
q \rho(x)=q(x) \rho \text { for all } x \in E \text {. }
$$

If $\rho$ is symmetric, then $K$ is an involution of $k$ of the first kind with trace space $l_{\rho}$, and $q \rho$ is a $K$-quadratic form on $E$ with polar form $f \rho$. Conversely if $q$ is non-zero and $q \rho$ is an $L$-quadratic form on $E$ for some involution $L$ of $k$, then $L=K$, so $K$ is an 
involution and so $\rho$ is symmetric.

2.2 Isometries. Suppose $k^{\prime}$ is a division ring with involution $J^{\prime}, E^{\prime}$ is a left vector space over $k^{\prime}$ and $q^{\prime}$ is a $J^{\prime}$-quadratic form on $E^{\prime}$ with polar form $f^{\prime}$. A semi-isomorphism $X: E^{\prime} \rightarrow E$ with isomorphism $\sigma: k^{\prime} \rightarrow k$ is called a semi-similarity from $\left(E^{\prime}, q^{\prime}\right)$ to $(E, q)$ if

$$
q^{X} \rho=q^{\prime}
$$

for some non-zero $\rho \in k^{\prime}$. Provided $q$ is not the zero form, the element $\rho$ is unique and we call it the multiplier of $X$. By (2.1) the multiplier is symmetric and is related to $\sigma$ by

$$
\lambda^{J^{\prime}}=\rho^{-1} \lambda^{\sigma J \sigma^{-1}} \rho \text { for all } \lambda \in k^{\prime} \text {. }
$$

Suppose now that $k^{\prime}=k$. A semi-similarity form $\left(E^{\prime}, q^{\prime}\right)$ to $(E, q)$ with identity automorphism of $k$ is called a similarity, and a similarity with trivial multiplier is called an isometry. We say that the forms $q$ and $q^{\prime}$ are equivalent if there exists an isometry from $\left(E^{\prime}, q^{\prime}\right)$ to $(E, q)$.

2.3 Unitary groups. Define $\Gamma U(E, q)$ to be the set of all semi-similarities of $(E, q)$, define $G U(E, q)$ to be the set of all similarities of $(E, q)$, and define $U(E, q)$ to be the set of all isometries of $(E, q)$. Then it is easily verified that $\Gamma U(E, q)$ is a subgroup of the full semi-automorphism group $\Gamma L(E)$ of $E$, and that $G U(E, q)$ and $U(E, q)$ are normal subgroups of $\Gamma U(E, q)$.

We call an isometry $X$ of $(E, q)$ finite dimensional if the space $E(I+X)$ is finite dimensional, and define $U_{\varphi}(E, q)$ to be the set of all finite dimensional isometries of $(E, q)$. Then again $U_{\varphi}(E, q)$ is a normal subgroup of $r U(E, q)$.

We denote the images of these groups in the full collineation group $P \Gamma L(E)$ of the projective space $P(E)$ respectively by $P \Gamma U(E, q)$, $P G U(E, q), P U(E, q)$ and $P U_{\varphi}(E, q)$. Every homothety $\lambda I$ of $E$, with $\lambda \in k^{\#}$, is clearly a semi-similarity of $(E, q)$ with multiplier $\left(\lambda^{J} \lambda\right)^{-1}$. Hence the intersection of $U(E, q)$ with the group of 
homotheties of $E$ is trivial, and we may regard $U(E, q)$ as being faithfully represented on the projective space $P(E)$.

Suppose $q$ is non-degenerate with defect space the whole of $s / 2$, and let $(\bar{E}, \bar{f})$ be the space $E / R(E)$ with the canonical non-degenerate image of $f$. Then it is straightforward to show that the canonical epimorphism from $E$ to $\bar{E}$ induces an isomorphism

$$
\psi: \Gamma U(E, q)+\Gamma U(\bar{E}, \bar{f})
$$

carrying $G U(E, q), U(E, q)$ and $U_{\varphi}(E, q)$ onto $G U(\bar{E}, \bar{f}), U(\bar{E}, \bar{f})$ and $U_{\varphi}(\bar{E}, \bar{f})$ respectively. This and Lemma 1.5 show that unitary groups of $J$-hermitian forms over $k$ may be regarded alternatively as unitary groups of $J$-quadratic forms over $k$ of maximal defect ( $c f$. Dieudonné [5], p. 35$)$.

If $\rho \in k^{\#}$ is any symmetric element, then $q \rho$ is a $\rho^{-1} J \rho$-quadratic form and the various unitary groups of ( $E, q p)$ are equal to the corresponding unitary groups of $(E, q)$. Now if $k$ is not a field and $\rho=\lambda+\lambda^{J} \in l^{\#}$, then $l$ is a trace with respect to $\rho^{-1} J \rho$, since $\rho^{-1} \lambda+\rho^{-1}\left(\rho^{-1} \lambda\right)^{J} \rho=1$, hence we may replace $q$ by $q \rho$ and assume that $l$ is a trace. Also when $k$ is a field and $q$ is defective, we may assume that 1 lies in the defect space of $q$.

2.4 Cayley parametrization. In this section we construct the usual parametrization of the finite-dimensional isometries of $(E, q)$ by means of Cayley spaces and forms. We shall assume the discussion of the process for sesquilinear forms given in $W a l l[11]$ and adapt it to J-quadratic forms in the same way as is done there for quadratic forms over fields.

Suppose that $X$ is an isometry of $(E, q)$ and put $X=1+N$. We define the Cayley space and form $\left(E_{X}, f_{X}\right)$ of $X$ to be the Cayley space and form of $X$ with respect to the polar form $f$. We recall then from [11] that $E_{X}$ is the image $E N$ of $N$, and $f_{X}$ is the $J$-sesquilinear form on $E_{X}$ uniquely defined by

$$
f_{X}(x N, y N)=f(x, y N) \text { for all } x, y \in E \text {. }
$$


Then the Cayley form satisfies

$$
f_{X}(u, v)+f_{X}(v, u)^{J}=f(u, v) \text { for all } u, v \in E_{X} \text {. }
$$

If the Cayley space $E_{X}$ is disjoint from $R(E)$, then the Cayley form $f_{X}$ is non-degenerate and the fixed point space kerN of $X$ is $E_{X}^{\perp}$. If $E_{X}$ is finite dimensional and disjoint from $R(E)$, and if $\left(e_{1}, \ldots, e_{r}\right)$ is any basis of $E_{X}$ and $\left(\theta_{i j}\right)$ is the inverse of the matrix $\left(f\left(e_{i}, e_{j}\right)\right)$ of $f_{X}$ with respect to this basis, then the isometry $X$ can be written

$$
X=1+\sum_{i, j=1}^{r} e_{i} \theta_{i j}{ }_{j}
$$

meaning

$$
x X=x+\sum_{i, j=1}^{r} f\left(x, e_{i}\right) \theta_{i j} e_{j} \text { for all } x \in E \text {. }
$$

In our case $X$ satisfies the further condition that $q(x X)=q(x)$ for all $x \in E$, and so $f(x, x N) \in q(x N)$ for all $x \in E$. So the Cayley form satisfies

$$
f_{X}(u, u) \in q(u) \text { for all } u \in E_{X} \text {. }
$$

Suppose $x N \in R(E)$. Then $f(x, x N)=0$ and so $q(x N)=0$ and $x N \in K(E)$. We conclude that

$$
E_{X} \cap R(E)=E_{X} \cap K(E) \text {. }
$$

In particular if $q$ is non-degenerate then the Cayley space of $X$ is disjoint from $R(E)$.

LEMMA 2.5. (a) Suppose $X$ is an isometry of $(E, q)$ with Cayley space and form $(V, g)$. Then

$$
\begin{gathered}
g(u, v)+g(v, u)^{J}=f(u, v) \text { for } a z z, v \in V, \\
g(v, v) \in q(v) \text { for alz } v \in V, \\
V \cap R(E)=V \cap K(E) .
\end{gathered}
$$


(b) Conversely suppose $g$ is a non-degenerate J-sesquilinear form on a finite dimensional subspace $V$ of $E$ disjoint from $R(E)$ satisfying (2). Then there exists a unique isometry of $(E, q)$ with Cayley space and form $(v, g)$.

(c) Suppose $g$ is a J-sesquilinear form on a subspace $V$ of $E$ with basis $\left\{e_{i} \mid i \in I\right\}$ satisfying

$$
\begin{gathered}
g\left(e_{i}, e_{j}\right)+g\left(e_{j}, e_{i}\right)^{J}=f\left(e_{i}, e_{j}\right) \text { for all } i, j \in I, \\
g\left(e_{i}, e_{i}\right) \in q\left(e_{i}\right) \text { for all } i \in I .
\end{gathered}
$$

Then $g$ satisfies (1) and (2).

Proof. (b) We first show that $g$ satisfies ( 1 ). This follows easily from (2) when $k$ is a field, so we assume $k$ is not a field. From (2) it follows that

$$
\operatorname{Tr}(g(v, v))=f(v, v) \text { for all } v \in V .
$$

If $u, v \in V$ and $\lambda, \mu \in k$ then substituting $\lambda u+\mu v$ in this equation gives

$$
\operatorname{Tr}\left(\lambda g(u, v) \mu^{J}+\mu g(v, u) \lambda^{J}\right)=\operatorname{Tr}\left(\lambda f(u, v) \mu^{J}\right)
$$

and so

$$
\lambda\left(f(u, v)+g(u, v)+g(v, u)^{J}\right) \mu^{J} \in \mathbf{s} .
$$

Condition ( 1 ) now follows easily. Hence there is a unique isometry $X$ of $(E, f)$ with Cayley space and form $(V, g)$ (see Wall [11]), and we must show that $X$ preserves $q$.

Suppose $x \in E$ and put $X=1+N$. Then

$$
\begin{aligned}
q(x X) & =q(x)+q(x N)+(f(x, x N)+Z) \\
& =q(x)+(g(x N, x N)+Z)+(g(x N, x N)+Z) \\
& =q(x) .
\end{aligned}
$$

(c) Condition ( 1 ) follows easily by $J$-sesquilinearity, and the proof of (2) involves a straightforward expansion of $q\left(\sum_{i \in F} \lambda_{i} e_{i}\right)$ where $F$ is a finite subset of $I$ and $\lambda_{i} \in k$ for all $i \in F$. 
LEMMA 2.6. Suppose $X$ is a finite-dimensional isometry of $(E, q)$ with cayley space and form $(V, g)$ such that $V$ is disjoint from $R(E)$. Suppose $\left(e_{1}, \ldots, e_{r}, \ldots, e_{s}\right)$ is a basis of $V$ with respect to which $g$ has the partitioned form

$$
[g]=\left[\begin{array}{c:c}
A & B \\
\hdashline 0 & C
\end{array}\right] \text {. }
$$

Then $X$ is the product $Y Z$, where $Y$ and $Z$ are the isometries of $(E, q)$ whose Cayley spaces are respectively $\left(e_{1}, \ldots, e_{p}\right)$ and $\left(e_{r+1}, \ldots, e_{s}\right)$ and whose forms are the corresponding restrictions of $g$.

2.7 One-dimensional isometries. Suppose $a \in E \backslash R(E)$. Then the isometries of $(E, q)$ with Cayley space $(a)$ are all the isometries of the form

$$
1+a \alpha^{-1} a
$$

where $\alpha \in q(\alpha)$ is non-zero. If $k$ is a field, then either $a$ is singular and there is no isometry with Cayley space $(a)$, or $a$ is non-singular and there is a unique isometry $1+a q(a)^{-1} a$ with Cayley space $\langle a\rangle$. If $k$ is not a field, then there are infinitely many isometries with Cayley space $\langle a\rangle$, since the trace space $l$ is infinite.

Suppose $P=1+a \alpha^{-1} a$ is a one-dimensionel isometry of $(E, q)$, where $a \in E \backslash R(E)$ and $\alpha \in q(a)$ is non-zero. If $a$ is isotropic then $P$ is a transvection of $E$ and we call $P$ a transvection of $(E, q)$. If $a$ is non-isotropic then $P$ is a dilatation of $E$ and we call $P$ a quasi-symetry of $(E, q)$. Clearly there are quasi-symmetries of $(E, q)$ if and only if $k$ is not a field and $f$ is non-zero.

WITT'S THEOREM 2.8. Suppose that $V$ and $w$ are finite dimensional subspaces of $E$ disjoint from $R(E)$ and that $X: V+W$ is an isometry from $\left(V,\left.q\right|_{V}\right)$ to $\left(\omega,\left.q\right|_{W}\right)$. Then $X$ can be extended to a finite dimensional isometry of $(E, q)$.

Proof (cf. Wall [11], proof of 1.2.1). We prove the theorem by induction on the dimension of $V$. Choose $x \in V^{\#}$ and choose $V_{1} \leq V$ so 
that $V=V_{1} \oplus\langle x)$. Then $w=V_{1} X \oplus\langle x X)$, and by the induction hypothesis we may assume that $v_{1} X=v_{1}$ and that $X$ acts trivially on $v_{1}$

Suppose $x X \neq x$ (or else the identity map on $E$ extends $X$ ) and put $y=x+x X$. Then

$$
\begin{gathered}
y \in v_{1}^{L}, \\
f(x, y)+f(y, x)=f(y, y), \\
f(x, y) \in q(y) .
\end{gathered}
$$

Suppose $f(x, y) \neq 0$. Since $f(x, y) \in q(y)$ we may define an isometry $T$ of $(E, q)$ by

$$
T=1+y f(x, y)^{-1} y,
$$

and then clearly $T$ extends $X$.

Suppose $f(x, y)=0$; then $q(y)=0$. If $y \in K(E)$, choose $u$ a complement of $(x)$ in $E$ containing $V_{1}$ and define an isometry $T$ of $(E, q)$ by

$$
\begin{gathered}
x T=x+y, \\
u T=u \text {, for all } u \in u .
\end{gathered}
$$

So we assume $y \leqslant K(E)$, and so $y \leqslant R(E)$.

If $y \leqslant V$ then $x$ and $y$ are independent modulo $V_{I}$, so we can choose $z \in V_{1}^{\perp}$ so that $f(x, z)=1$ and $f(y, z) \neq 1$. If $y \in V$ then $y$ does not lie in the coset $x+V_{1}$ since $x X \vDash V_{1}$, so we choose $z \in V_{1}^{\perp}$ so that $f(x, z)=1$ and then $f(y, z) \neq 1$. Choose $\lambda \in q(z)$ and then

$$
\left[\begin{array}{cc}
0 & 1 \\
f(z, y)+1 & \lambda
\end{array}\right]+\left[\begin{array}{cc}
0 & 1 \\
f(z, y)+1 & \lambda
\end{array}\right]^{J \cdot t}=\left[\begin{array}{ll}
f(y, y) & f(y, z) \\
f(z, y) & f(z, z)
\end{array}\right] .
$$

Also $0 \in q(y), \lambda \in q(z)$, and the first matrix is non-degenerate. So there is a unique isometry $T$ of $(E, q)$ whose Cayley space is $(y, z)$ and whose Cayley form is given by the first matrix above with respect to 
the basis $(y, z)$. Taking the inverse of this matrix and using $f(x, y)=0, f(x, z)=1$ gives

$$
\begin{gathered}
v_{1} T=v_{1} \text { for all } v_{1} \in v_{1}, \\
x T=x+y,
\end{gathered}
$$

and so $T$ extends $X$.

COROLLARY 2.9. Every finite-dimensional singular subspace of $(E, q)$ disjoint from $R(E)$ is contained in a singular subspace of $(E, q)$ disjoint from $R(E)$ of dimension equal to the witt index of $q$.

THEOREM 2.10. Suppose $q$ is non-degenerate and $f$ is not the zero form.

(a) The centralizer of $U_{\varphi}(E, q)$ in $\Gamma L(E)$ is the group of homotheties of $E$, except for $O^{(1)}(2,2), O^{(1)}(2,4)$ and $O(3,2)$.

(b) The centre of $U_{\varphi}(E, q)$ is trivial, except for $O^{(1)}(2,2)$.

Proof. Every isometry of $(E, q)$ is k-linear and so commutes with every homothety of $E$. Conversely suppose $X$ is a semi-automorphism of $E$ centralizing $U_{\varphi}(E, q)$. Then $X$ commutes with every one-dimensional isometry of $(E, q)$ and so fixes every line $\langle x\rangle$ of $E$ outside $R(E)$ such that $q(x)$ contains a non-zero scalar. When $q$ is definite or $k$ is not a field, then $X$ fixes every line of $E$ outside $R(E)$, so as $R(E)<E, X$ fixes every line of $E$ and is a homothety. When $k$ is not $\mathrm{GF}(2)$, every hyperbolic plane contains at least two non-singular lines and so is fixed by $X$, hence if also $n \geq 3$ and $q$ is indefinite, $X$ fixes every line of $E$ outside $R(E)$ by Lemma 1.10. We shall omit the remaining cases (see Dieudonné [5], p. 63).

Part (b) follows now since the intersection of $U_{\varphi}(E, q)$ with the group of homotheties is trivial, except in the three exceptional cases which we shall omit.

LEMMA 2.11. Suppose $g$ is a non-degenerate $J$-sesquizinear form on a left vector space $V$ of finite dimension $r$ over $k$.

(a) If $k$ is a field but not GF (2), then either $g$ is an 
alternating form or $U$ has a basis upper triangularizing the matrix of $g$.

(b) If $k$ is not a field, then $V$ has a basis upper triangularizing the matrix of $g$, and unless $g(x, x)$ is symmetric for all $x \in V$, this basis can be so chosen that every diagonal element is non-symmetric.

Proof. (l) First we prove by induction on the dimension of $V$, assuming that $k$ is not $\mathrm{GF}(2)$, that if $g$ is non-alternating then the matrix of $g$ can be upper triangularized. Suppose by way of contradiction that $g$ is non-alternating but cannot be upper triangularized. Then if $x$ is any non-isotropic vector of $V$, it follows by the induction hypothesis that the restriction of $g$ to the $(r-1)$-space left orthogonal to $x$ is alternating and that this space is non-zero (and hence $J=1$ ). Choose $a \in V$ non-isotropic; then we can choose a hyperbolic pair $(b, c)$ in the space left orthogonal to $a$, and the matrix of the restriction of $g$ to the 3-space with basis $(a, b, c)$ has the form

$$
\left[\begin{array}{lll}
\alpha & \beta & \gamma \\
0 & 0 & 1 \\
0 & 1 & 0
\end{array}\right] \text {, where } \alpha \neq 0 \text {. }
$$

We can choose $b$ both left and right orthogonal to $a$ and replace $a$ by any non-zero multiple of $a$, so we can assume that $\beta=0$ and (since $k$ is not GF(2)) that $\gamma \neq \alpha$. Put $x=a+\alpha b$ and $y=a+c$, then $x$ and $y$ are both non-isotropic but $y$ is left orthogonal to $x$, a contradiction.

(2) We prove the second part of (b) by induction on the dimension of $V$. Suppose then that $V$ contains vectors $x$ so that $g(x, x)$ is non-symmetric, but $g$ cannot be upper triangularized so that every diagonal element is non-symmetric. Then if $x$ is any vector of $V$ so $g(x, x)$ is non-symmetric, it follows by the induction hypothesis, that if $y$ is any vector in the $(x-1)$-space left orthogonal to $x$ then $f(y, y)$ is symmetric, and that this $(r-1)$-space is non-zero. Choose $a \in V$ so $g(a, a)$ is non-symetric; then we can choose $b$ non-isotropic in the space left orthogonal to $a$, and the matrix of the restriction of $g$ to the 2-space with basis $(a, b)$ has the form

$$
\left[\begin{array}{ll}
\alpha & \gamma \\
0 & \beta
\end{array}\right] \text {, where } \beta \in s^{\#} \text { and } \alpha \neq s \text {. }
$$


We can replace $b$ by any non-zero multiple of $b$, so we can assume that $\beta \neq 1$ (if $\beta=1$ choose $\mu \in k^{\#}$ so that $\mu \mu^{J} \neq 1$, and replace $b$ by $\mu b)$. We can replace $a$ by any non-zero multiple of $a$, so we can assume that $\gamma=0$ or $\gamma=1$. If $\gamma=0$ put $x=a+B^{-1} b$ and $y=a+\alpha b$, then both $g(x, x)$ and $g(y, y)$ lie in $\alpha+s$ and $y$ is left orthogonal to $x$. So we assume that $\gamma=1$.

Suppose $\lambda \in k^{\#}$ and put $x=a+\lambda b$ and $y=a+\mu b$ where $\mu=\left(\alpha\left(\lambda^{-1}\right)^{J}+1\right) \beta^{-1}$. Then $y$ is left orthogonal to $x$ and

$$
\begin{gathered}
g(x, x) \in\left(\alpha+\lambda^{J}\right)+s, \\
g(y, y) \in\left(\alpha+\alpha\left(\lambda^{-1}\right)^{J_{B}-1}\right)+s,
\end{gathered}
$$

where we have used $\xi \in \xi^{J}+8$ for all $\xi \in k$. First put $\lambda=\alpha^{J} \beta^{-1}$. Then $g(y, y)$ is non-symmetric, hence $\alpha+\beta^{-1} \alpha$ is symmetric and so

$$
\left(1+\beta^{-1}\right) \alpha=\alpha^{J}\left(1+\beta^{-1}\right) \text {. }
$$

Secondly, put $\lambda=1$. Then $g(x, x)$ is non-symmetric, hence $\alpha+\alpha \beta^{-1}$ is symmetric and so

$$
\alpha\left(1+\beta^{-1}\right)=\left(1+\beta^{-1}\right) \alpha^{J}
$$

Combining these gives the result that $\alpha$ commutes with $I+B^{-2}$. Thirdly, put $\lambda=\beta$. Then $g(x, x)$ is non-symmetric hence $\alpha+\alpha \beta^{-2}$ is symmetric. But $\alpha$ commutes with $\left(1+\beta^{-2}\right)$, so $1+\beta^{-2}=0$ and $\beta=1$. This contradicts the construction of $b$.

THEOREM 2.12 (ef. Dieudonné [4]). Suppose $q$ is non-degenerate, and suppose $X$ is a non-trivial isometry of $(E, q)$ with Cayley space $V$ of finite dimension $r$.

(a) If $k$ is not a fiezd; then either $V$ is non-isotropic and $X$ is the product of $r$ quasi-symmetries of $(E, q)$ and no fewer, or $V$ is isotropic and $X$ is the product of $r+2$ quasi-symmetries and no fewer.

(b) (Dieudonné [4], Theorems 6 and 7). (i) If $k$ is a field but 
not $G F(2)$, then either $V$ is non-singular and $X$ is the product of $r$ transvections of $(E, q)$ and no fewer, or $V$ is singular and $X$ is the product of $r+2$ transvections and no fewer.

(ii) If $k$ is $G F(2)$ then $X$ is the product of transvections of $(E, q)$, except that if $n=4$ and $v=2$ then $X$ is the product of transvections and 2-transvections with singular Cayley space.

Proof. (a) If $V$ is isotropic, choose $x \in E$ non-isotropic, choose $\alpha \in q(x)$ and put $P=1+x \alpha^{-1} x$. Then the Cayley space of $X P$ is $V \oplus\langle x\rangle$ of dimension $r+1$, so we can assume $V$ is non-isotropic. Then the Cayley space $V$ of $X$ contains vectors $v$ so that $g(v, v)$ is not symmetric (where $g$ is the Cayley form of $X$ ), and so by Lemma 2.11 we can choose a basis $\left(e_{1}, \ldots, e_{p}\right)$ of $V$ upper triangularizing $g$ so that $g\left(e_{i}, e_{i}\right)$ is not symmetric, for all $i$. Now by Lemma 2.6,

$$
X=\left(1+e_{1} g\left(e_{1}, e_{1}\right)^{-1} e_{1}\right) \ldots\left(1+e_{r} g\left(e_{r}, e_{p}\right)^{-1} e_{r}\right)
$$

is the product of $r$ quasi-symmetries.

(b) Part (b) is proven in Dieudonne [4] (the case when $k$ is not $G F(2)$ follows easily from the previous lemma).

\section{A simple subgroup of the unitary group}

Suppose that the division ring $k$ and the vector space $E$ are as in the first section. Suppose that $q$ is a non-degenerate indefinite $J$-quadratic form on $E$ of Witt index $v$ with polar form $f$ and defect space $m$, and if $k$ is a field suppose that $E$ has dimension at least 3 . Suppose that $l$ is a trace when $k$ is not a field and that 1 lies in the defect space when $q$ is defective and $k$ is a field.

We shall define a normal subgroup of $\Gamma U(E, q)$ inside $U_{\varphi}(E, q)$ which coincides with the commutator subgroup $\Omega(E, q)$ when $k$ is a field (except for $O(3,2), O^{(2)}(4,2)$ and $\left.O(5,2)\right)$, and prove the simplicity of this group (except for $O(3,2), O(5,2)$ and $o^{(2)}(4, k)$ when $k$ is a field).

3.1 Transvections and quasi-transvections. We recall that an 
r-tranovection of $E$ is an automorphism $P$ of $E$ whose Cayley space has finite dimension $r$ and is centralized by $P$, and that transvections are 1-transvections. A singular $p$-transvection of $(E, q)$ is an r-transvection of $(E, q)$ whose Cayley space is singular. Thus a singular transvection $P$ can be written

$$
P=1+e \alpha^{-1} e \text {, }
$$

where $e \in E^{\#}$ is singular and $\alpha \in l^{\#}$. Clearly $(E, q)$ has singular transvections if and only if $k$ is not a field.

A semi-singular p-transvection of $(E, q)$ is an r-transvection of $(E, q)$ whose Cayley space is semi-singular. Thus a semi-singular transvection $P$ can be written

$$
P=1+(e+a) \alpha^{-1}(e+a),
$$

where $e \in E^{\#}$ is singular, $\alpha \in R(E)$ and $\alpha \in q(\alpha)$ is non-zero. Every singular transvection is a semi-singular transvection, and $(E, q)$ has non-singular semi-singular transvections if and only if $q$ is defective.

A quasi-transvection of $(E, q)$ is a two-dimensional isometry $P$ of $(E, q)$ of the form

$$
P=1+e \alpha e+e a+a e,
$$

where $e \in E^{\#}$ is singular, $a \in e^{\perp}$ is isotropic and independent modulo $R(E)$ of $e$, and $\alpha \in q(a)$. Thus a quasi-transvection is a 2-transvection of $(E, q)$ and has order 2 . Clearly $(E, q)$ has quasi-transvections if and only if the Witt index of $f$ is at least 2 .

If $P$ is a semi-singular transvection of $(E, q)$, then by replacing $e$ by $\alpha e$ in the expression for $P$ above, we can write $P$ as

$$
P=1+e a e+e a+a e,
$$

where $e \in E^{\#}$ is singular, $a \in R(E)$ and $\alpha \in q(a)$ is non-zero.

If $k$ is a field then every singular 2-transvection is a quasi-transvection, but when $k$ is not a field there may be singular 2-transvections which are not quasi-transvections.

DEFINITION 3.2. The set of singular transvections, the set of 
semi-singular transvections, and the set of quasi-transvections is each a normal subset of $\Gamma U(E, q)$ and so each generates a normal subgroup of $\Gamma U(E, q)$ inside $U_{\varphi}(E, q)$. We define the normal subgroup $T(E, q)$ of $\Gamma U(E, q)$ to be the subgroup of $U_{\varphi}(E, q)$ generated by all singular transvections, semi-singular transvections and quasi-transvections of $(E, q)$. Once it is shown that $T(E, q)$ is simple it will follow that each of the three sets is either empty or generates $T(E, q)$. However, the proof of this need not be so indirect; in particular quasitransvections are fairly readily shown directly to be products of semi-singular transvections whenever these last exist. Our definition is intended to show the unity amongst the various cases.

LEMMA 3.3. (a) If an automorphism $X$ of $E$ fixes every singular line of $(E, q)$ then $X$ is a central homothety, except when $k$ is quaternionic and $n=2$.

(b) The group $T(E, q)$ is faithfully represented as a permutation group on the set of singular lines of $(E, q)$.

Proof. (a) If $k$ is $G F(2)$ then by Lemma 1.6, $X$ fixes a basis of $E$ and so is trivial. If $k$ is not $\mathrm{GF}(2)$ and $n \geq 3$, then since $X$ fixes every hyperbolic plane of $(E, q), X$ fixes every line of $E$ outside $R(E)$ by Lemma 1.9 and so $X$ fixes every line of $E$ and is a central homothety. So we assume $n=2$ and so $k$ is not a field.

Choose a hyperbolic basis $(e, d)$ of $(E, q)$; then a line $(e+\lambda d\rangle$ where $\lambda \in k$ is singular if and only if $\lambda \in Z$. Since $X$ fixes both $\langle e\rangle$ and $\langle d\rangle$ we may put

$$
\begin{aligned}
& e X=\alpha e, \\
& d X=B d,
\end{aligned}
$$

where $\alpha, \beta \in k^{\#}$. For each $\lambda \in Z, X$ fixes the line $(e+\lambda d)$ and so $\beta=\lambda^{-1} \alpha \lambda$. We have assumed 1 is a trace and so $\alpha=\beta$, and thus $\alpha$ commutes with every trace of $k$. Now when $k$ is not quaternionic, the traces generate $k$ and so $\alpha$ lies in the centre of $k$ as required. However when $k$ is quaternionic then $l$ coincides with $z$ and the condition is vacuous, and in fact every automorphism of $E$ defined by 
$e \rightarrow \alpha e$ and $d \rightarrow \alpha d$, where $\alpha \in k^{\#}$, fixes every singular line of $(E, q)$.

(b) We assume $n=2$ and $k$ is quatemionic, for otherwise the result follows from the first part. Choose a hyperbolic basis $(e, d)$ of $(E, q)$; then every singular transvection $P$ of $(E, q)$ has the form

$$
1+(\lambda e+\mu d) \zeta(\lambda e+\mu d),
$$

where $\lambda, \mu \in z$ and $\zeta \in z^{\#}$. Now

$$
\begin{aligned}
& e P=(1+\mu \zeta \lambda) e+\mu \zeta \mu d, \\
& d P=\lambda \zeta \lambda e+(1+\lambda \zeta \mu) d,
\end{aligned}
$$

and so $T(E, q)$ fixes the set of all z-linear combinations of $e$ and $d$. Hence again by the first part, the only isometry of $(E, q)$ in $T(E, q)$ fixing every singular line of $(E, q)$ is the identity. (This argument is a very special case of the Wall norm to be introduced in the next chapter.)

LEMMA 3.4. (a) Suppose $(e, d)$ is a hyperbolic pair in $(E, q)$, suppose $a \in\langle e, d\rangle^{\perp}$ is isotropic (possibly zero) and suppose $\alpha \in q(a)$ is non-zero. Then $T(E, q)$ contains the isometry $X$ of $(E, q)$ defined by

$$
\begin{gathered}
e X=\alpha d, \\
d X=\alpha^{-1} e, \\
x X=x+(x, a) \alpha^{-1} a \text { for all } x \in\langle e, d\rangle^{2} .
\end{gathered}
$$

(b) Suppose $\lambda \in k^{\#}$ is a product of an even number of elements $\alpha \in k^{\#}$ such that $\alpha \in q(a)$ for some isotropic vector $a \in(e, d)^{+}$. Then $T(E, q)$ contains the isometry with Cayley space $\langle e, d\rangle$ defined by

$$
\begin{gathered}
e X=\lambda e, \\
d X=\left(\lambda^{-1}\right)^{J} d .
\end{gathered}
$$

(Note that the restriction "even number" is relevant only when $k$ is a field and $q$ is nondefective, for otherwise we have assumed that $I$ lies in the defect space.) 
Proof. It will suffice to prove the first part. Define isometries $P$ and $Q$, generators of $T(E, q)$ by

$$
\begin{gathered}
P=1+e \alpha^{-1} e+e\left(\alpha^{-1} a\right)+\left(\alpha^{-1} a\right) e, \\
Q=1+d a d+d a+a d .
\end{gathered}
$$

Then $P Q P$ is the required isometry in $T(E, q)$.

LEMMA 3.5. (a) Suppose $V$ and $w$ are singular subspaces of $(E, q)$ so that $V+W$ is non-degenerate with respect to $f$. Then every transvection of $V$ can be extended to a singular 2-transvection of $(E, q)$ with Cayley space in $V+W$.

(b) Every automorphism of $V$ in the group $S L(V)$ generated by tronsvections of $V$ can be extended to an isometry in $T(E, q)$ with Cayley space in $V+W$.

Proof. It will suffice to prove the first part. Suppose $T=1+\varepsilon e$ is a transvection of $V$, where $e \in V^{\#}$ and $\varepsilon$ is a non-zero linear form on $V$ annihilating $e$. Choose $d \in W$ so that for all $v \in V$, $f(v, d)=(v, \varepsilon)$. Then the singular 2-transvection $P=1+e d+d e$ extends $T$.

LEMMA 3.6. (a) For every finite $r \leq v$, the group $T(E, q)$ acts faithfully on the set of singular r-subspaces of $(E, q)$.

(b) For every finite $r \leq v$, the group $T(E, q)$ acts transitively on the set of singular p-subspaces of $(E, q)$, except possibly when $r=v$ and $n=2 v$ and $k$ is a field (in this case the set divides into two orbits under $T(E, q)$, see Dieudonné [5]).

(c) For every finite $r \leq v-1$, every isomorphism between two singular r-subspaces of $(E, q)$ can be extended to an isometry in $T(E, q)$.

Proof. (a) Suppose $X \in T(E, q)$ fixes every singular $r$-subspace of $(E, q)$, and suppose $e \in E^{\#}$ is singular. Extend $e$ to a basis $\left(e_{1}=e, e_{2}, \ldots, e_{r}\right)$ of a singular $r$-subspace of $(E, q)$ containing $e$, and choose a complementary set $\left(d_{1}, \ldots, d_{r}\right)$ according to Lemma 1.6; then $(e)$ is the intersection of the two singular r-subspaces 
$\left\langle e_{1}, \ldots, e_{p}\right\rangle$ and $\left\langle e, d_{2}, \ldots, d_{p}\right\rangle$ of $(E, q\rangle$ and so is fixed by $X$. So $X$ fixes every singular line of $(E, q)$ and so by 3.3 is trivial.

(b) We prove transitivity by induction on $r$. Suppose $V$ and $W$ are singular $p$-subspaces of $(E, q)$; then by the induction hypothesis we may assume that $V \cap W$ has dimension $r-1$ and put

$$
\begin{aligned}
& v=\left(V_{n} w\right) \oplus\langle e\rangle, \\
& w=\left(v_{n} w\right) \oplus\langle d\rangle .
\end{aligned}
$$

If $e$ and $d$ are orthogonal, then $V+W$ is a singular $(r+1)$-space and the result follows by Lemma 3.5 (since for any vector space $E$, the transvection subgroup $S L(E)$ is transitive on the set of all r-subspaces of $E$, for all finite $r \leq$ dimE). If $e$ and $d$ are not orthogonal then the result follows by Lemma 3.4, provided that either the trace space is non-zero, or we can choose an isotropic non-singular vector $a$ in $(v+w)^{\perp}$. This is always possible except when $k$ is a field, $r=v$ and $n=2 v$.

(c) Choose $X \in T(E, q)$ so that $V X=W$ and choose a singular $(p+1)$-subspace $U$ of $(E, q)$ containing $W$. Now the result follows by Lemma 3.5, since for any vector space $E$ every isomorphism between two $r$-subspaces of $E$ can be extended to an automorphism in the transvection subgroup $S L(E)$, for all finite $r \leq \operatorname{dim} E-1$.

LEMMA 3.7. (a) The stabilizer in $T(E, q)$ of a singular line $\langle e\rangle$ of $(E, q)$ is transitive on the singular lines of $(E, q)$ not orthogonal to $i t$.

(b) Suppose $v \geq 2$, and if $k$ is a field suppose $n \geq 5$. Then the stabilizer in $T(E, q)$ of a singular line $(e\rangle$ of $(E, q)$ is transitive on the remaining singular lines of $(E, q)$ orthogonal to it.

(c) The group $T(E, q)$ acts doubly transitively on the singular lines of $(E, q)$ if and only if $\nu=1$. Unless perhaps $k$ is a field and $n=4$ and $v=2$, the grow $T(E, q)$ acts primitively on the singular lines of $(E, q)$.

Proof. (a) Suppose $\langle x\rangle$ and $\langle y\rangle$ are distinct singular lines of $(E, q)$ not orthogonal to $(e)$, and suppose $f(e, x)=f(e, y)=1$. Then 
$x+y \in e^{l}$ and $f(x, y) \in q(x+y)$, so we may define the isometry

$$
P=1+e f(x, y) e+e(x+y)+(x+y) e,
$$

a generator of $T(E, q)$ ( $P$ is a singular transvection if $x+y \in\langle e\rangle$, a semi-singular transvection if $x+y \in\langle e\rangle+R(E)$, and a quasitransvection otherwise). Now $e P=e$ and $x P=y$ as required.

(b) Suppose $\langle x\rangle$ and $\langle y\rangle$ are distinct singular lines of $(E, q)$ distinct from and orthogonal to $\langle e\rangle$. Choose $X \in T(E, q)$ such that $\langle e, x\rangle X=\langle e, y\rangle$ by Lemma 3.6. Choose an automorphism $T$ of $(e, y)$ in $S L\langle e, y\rangle$ such that $e X T=e$ and $x X T \in\langle y\rangle$, and extend by Lemma 3.5 to an isometry $Y$ of $(E, q)$ in $T(E, q)$. Then $X Y$ is the required isometry .

(c) It follows now that $T(E, q)$ is doubly transitive if and only if $v=1$. Suppose then $v \geq 2$, and suppose that $\Delta$ is a set of singular lines of $(E, q)$ of order at least 2 such that for all $X \in T(E, q)$,

$$
\Delta X=\Delta \text { or } \Delta X \cap \Delta=\emptyset \text {. }
$$

Choose $(e)$ in $\Delta$, choose $d \in E$ so $(e, d)$ is a hyperbolic pair, and choose $a \in(e, d)^{\perp}$ singular and non-zero.

If $\Delta$ contains a singular line not orthogonal to $\langle e\rangle$, then $\Delta$ contains all singular lines not orthogonal to $(e)$ by part $(a)$ and so contains $\langle d\rangle$. Again $\Delta$ contains all singular lines not orthogonal to $\langle d\rangle$ and so contains $\langle e+a\rangle$. But $\langle e+a\rangle$ is orthogonal to and distinct from $(e)$.

If $\Delta$ contains a singular line orthogonal to and distinct from $\langle e\rangle$, then $\Delta$ contains all such singular lines by part $(b)$ and so contains $\langle a\rangle$. Again $\Delta$ contains all singular lines orthogonal to $\langle a\rangle$ and so contains $(d)$. But $(d)$ is not orthogonal to $(e)$.

The order of $\Delta$ is at least two, so $\Delta$ contains all singular lines of $(E, q)$. So $T(E, q)$ acts primitively on the singular lines of $(E, q)$.

LEMMA 3.8. (a) Suppose $k$ is not $\mathrm{GF}(2)$. Then every singular transvection, non-singular semi-singular tronsvection and quasitransvection respectively is the product of two commuting singular 
tronsvections, non-singular semi-singular transvections or quasitronsvections respectively, conjugate in $T(E, q)$.

(b) The group $T(E, q)$ is perfect, except when $k$ is $\mathrm{GF}(2)$ and $n=3, n=5$, or $n=4$ and $v=2$.

Proof. (a) Let the isometry $P$ in question be written

$$
P=1+e \alpha e+e a+a e,
$$

where $e \in E^{\#}$ is singular, $a \in e^{l}$ is isotropic, $\alpha \in q(\alpha)$, and

$a=0$ and $\alpha \neq 0$ (singular transvection), or

$a \in R(E)^{\#}$ (non-singular semi-singular transvection), or

$a \notin\langle e, R(E)\rangle$ (quasi-transvection).

When $\alpha \neq 0$ or 1 , put $\lambda=\alpha$. When $\alpha=0$ or 1 and $k$ is not a field, choose $\lambda \in l^{\#}$ so that $\lambda \neq 1$. When $\alpha=0$ or $l$ and $k$ is a field, choose $\lambda \in k^{\#}$ so that $\lambda \neq 1$. By Lemma 3.4 we can choose $X \in T(E, q)$ so that

$$
\begin{aligned}
& e X=\lambda^{2} e, \\
& a X=a,
\end{aligned}
$$

(when $k$ is a field then $n \geq 3$, so we can choose $b \in e^{l}$ non-singular, then $\lambda^{2}=q\left(\beta^{-1} b\right) q(\lambda b)$ where $B=q(b)$; when $k$ is not a field then already $\lambda \in q(a))$. Put $\mu=\left(\lambda^{2}+1\right)^{-1} ;$ then $\mu \neq 0$ or 1 and $\lambda^{2} \mu+\mu=1$. Also $\lambda, \mu$ and $\alpha$ commute in pairs, and are symmetric.

Define the isometry $Q$ in $T(E, q)$ by

$$
Q=1+(\mu e) \alpha(\mu e)+(\mu e) a+a(\mu e),
$$

then

$$
Q^{X}=1+(1+\mu) e \alpha(1+\mu) e+(1+\mu) e a+\alpha(1+\mu) e .
$$

Now $Q$ and $Q^{X}$ commute, and are isometries of the same type as $P$ (singular transvections, etc). Finally, $Q Q^{X}=P$.

(b) When $k$ is not GF(2), the first part shows that every 
generator of $T(E, q)$ is itself a commutator of elements of $T(E, q)$, and so $T(E, q)$ is perfect. When $k$ is GF(2), the exhibition of the quasi-transvections and the semi-singular transvections as commutators of elements of $T(E, q)$ is more complicated, and we shall omit it.

THEOREM 3.9. The group $T(E, q)$ is simple, except possibly when $k$ is $\mathrm{GF}(2)$ and $n=3$ or $n=5$ and when $k$ is a field and $v=2$ and $n=4$ (when in fact $T(E, q)$ is not simple, see Dieudonné [5]).

Proof. By a lemma of Iwasawa [7] (see also Huppert [6]) a permutation group is simple if

(i) it is perfect, and

(ii) it is primitive, and

(iii) the stabilizer of a letter has a soluble normal subgroup whose normal closure in the whole group is the whole group.

Except in the cases excluded, we have proved that $T(E, q)$ is faithfully represented as a permutation group on the singular lines of $(E, q)$ (Lemma 3.3) and as such is primitive (Lemma 3.7) and perfect (Lemma 3.8).

Suppose $\langle e\rangle$ is a singular line of $(E, q)$ and let $N$ be the set of all isometries of $(E, q)$ of the form

$$
P(a, \alpha)=1+e \alpha e+e a+a e
$$

where $a \in e^{\perp}$ and $\alpha \in q(a)$. Then

$$
\begin{gathered}
P(a, \alpha) \cdot P(b, \beta)=P(a+b, \alpha+\beta+f(a, b)), \\
P(a, \alpha)^{-1}=P\left(\alpha, \alpha^{J}\right),
\end{gathered}
$$

and so $N$ is a subgroup of $U(E, q)$, clearly a normal subgroup of the stabilizer in $U(E, q)$ of $\langle e\rangle$. Furthermore

$$
[P(a, \alpha), P(b, \beta)]=P(0, \operatorname{Tr}(f(a, b))),
$$

and so the comnutator subgroup of $N$ is a subgroup of the abelian group of all singular transvections of $(E, q)$ with line $(e)$; so $N$ is soluble.

Hence $N \cap T(E, q)$ is a soluble normal subgroup of the stabilizer of (e) in $T(E, q)$ (in fact $N \leq T(E, q)$, but this fact is not necessary here). Since $T(E, q)$ is transitive on the singular lines of $(E, q)$, 
the normal closure of $N \cap T(E, q)$ in $T(E, q)$ is the whole of $T(E, q)$. So by Iwasawa's Lemma, $T(E, q)$ is a simple group. //

COROLLARY 3.10. The set of singular transvections, the set of non-singular semi-singular transvections, and the set of quasitransvections are each either empty or generating sets of $T(E, q)$.

Proof. The result is trivial when $T(E, q)$ is simple, and trivial in all the exceptional cases except when $k$ is $G F(2)$ and $n=5$. This case is easily settled by direct calculation.

\section{The Wall norm}

Suppose that the division ring $k$ and the vector space $E$ are as in the first section. Suppose that $q$ is a non-degenerate definite or indefinite $J$-quadratic form on $E$ of Witt index $v$ with polar form $f$ and defect space $m$. Suppose that $l$ is a trace when $k$ is not a field and that 1 lies in the defect space when $q$ is defective and $k$ is a field.

Denote by $\Delta$ the multiplicative group of the division ring $k$. Denote by $\Psi$ the subgroup of $\Delta$ generated by all non-zero norms $\lambda \lambda^{J}$ with $\lambda \in K^{\#}$; then $\Psi$ is normal in $\Delta$, since if $\mu \in \Delta$ then $\mu^{-1} \lambda \lambda^{J} \mu=\left(\mu^{-1} \lambda\right)\left(\mu^{-1} \lambda\right)^{J} \cdot \mu^{J} \mu \in \Psi$. Denote by $\Sigma$ the subgroup of $\Delta$ generated by the non-zero elements $\alpha \in k^{\#}$ such that $\alpha+z$ lies in the defect space of $q$; then $\Sigma$ is a normal subgroup of $\Delta$. Also $\Sigma$ contains $\Psi$, unless $k$ is a field and $q$ is non-defective, when $\Sigma$ is trivial.

Suppose that $w$ is any (possibly zero) vector of $E$, and denote by $\Omega_{w}$ the subgroup of $\Delta$ generated by all elements $\alpha \in k^{\#}$ such that $\alpha \in q(a)$ for some vector $a \in w^{\perp}$; then $\Omega_{w}$ is a normal subgroup of and contains $\Sigma$. Hence the commutator subgroup $\left[\Delta, \Omega_{\omega}\right]$ is normal in $\Delta$ and is contained in $\Omega_{w}$. When $q$ is indefinite, denote by $\Omega$ the subgroup of $\triangle$ generated by all $\alpha \in k^{\#}$ such that $\alpha \in q(a)$ for some vector $a \in \dot{E}$ orthogonal to some hyperbolic plane of $(E, q)$; then $\Omega$ 
coincides with $\Omega_{e}$ for every singular vector $e \in E^{\#}$.

We shall extend the norm introduced by Wall [10] to the present case and construct a homomorphism $N_{w}: U_{\varphi}(E, q) \rightarrow \Delta / \Sigma \Psi\left[\Delta, \Omega_{w}\right]$ which coincides with the spinor norm when $k$ is a field and $q$ is non-defective and with the Wall norm when the defect space is the whole of $s$; then we shall use it to determine the structure of the factor group of $U_{\varphi}(E, q)$ modulo $T(E, q)$. Much of the argument is identical to the corresponding argument in [10] and will be omitted.

$4.1 w$-bases. A w-basis of a finite dimensional subspace $V$ of $E$ is a basis $\left(e_{1}, \ldots, e_{p}\right)$ of $V$ satisfying:

for each $i=1, \ldots, r$ either $e_{i} \in w^{2}$ or $f\left(e_{i}, w\right)=1$.

LEMMA 4.2. Suppose that $P$ is a finite dimensional isometry of $(E, q)$ with Cayley space and form $(V, g)$. Suppose that $\left(a_{1}, \ldots, a_{p}\right)$ and $\left(b_{1}, \ldots, b_{r}\right)$ are two w-bases of $v$ upper triangularising $g$, so that

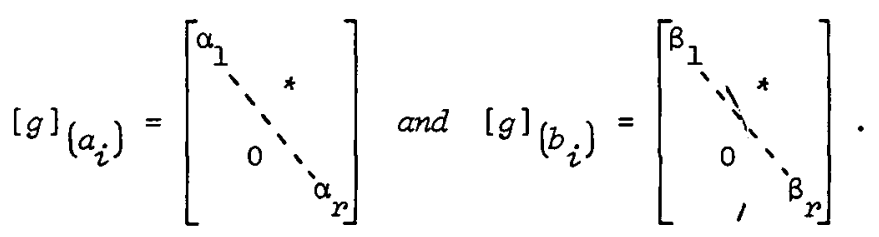

Then the elements $\alpha_{1}^{-1} \ldots \alpha_{r}^{-1}$ and $\beta_{1}^{-1} \ldots \beta_{r}^{-1}$ are equal modulo $\Psi\left[\Delta, \Omega_{\omega}\right]$.

Proof. When $k$ is a field, this follows from the invariance of the discriminant of $g$ modulo $\Psi$ (notice that in this case $\Delta / \Psi$ has exponent 2 , so the inverses in the last sentence are irrelevant).

When $k$ is not a field, the proof is identical, line by line, to the proof of the corresponding Lemma 5 in Wall [10].

DEFINITION 4.3. We define a map $N_{w}$ from $U_{\varphi}(E, q)$ to $\Delta / \Sigma \Psi\left[\Delta, \Omega_{w}\right]$ as follows: 
(i) define $N_{w}(1)=1$;

(ii) when $k$ is a field and $P \in U_{\varphi}(E, q)^{\#}$, define

$$
N_{w}(P)=(\operatorname{discrg}) \Sigma \Psi\left[\Delta, \Omega_{\omega}\right]
$$

(iii) when $k$ is not a field and $P \in U_{\varphi}(E, q)^{\#}$, define

$$
N_{w}(P)=f_{P}\left(a_{1}, a_{1}\right)^{-1} \ldots f_{P}\left(a_{r}, a_{p}\right)^{-1} \Sigma \Psi\left[\Delta, \Omega_{w}\right],
$$

where $\left(a_{1}, \ldots, a_{r}\right)$ is any w-basis of the Cayley space of $P$ upper triangularizing $f_{P}$.

Then by Lemma 4.2 and Lemma 2.11, $N_{w}$ is well-defined on every element of $U_{\varphi}(E, q)$. We call $N_{w}$ the Wall norm on $U_{\varphi}(E, q)$ with respect to $w$, and prove in the following theorem that $N_{w}$ is a homomorphism. (When $k$ is a field and $q$ is non-defective it is well known that there is another norm involved, the Dickson norm $D: O_{\varphi}(E, q)+Z_{2}$. For every $P \in O_{\varphi}(E, q), D(P)$ is defined to be the parity of the Cayley dimension of $P$; then $D$ is an epimorphism whose kernel is denoted by $S O(E, q)$. The following proof that $N_{w}$ is a homomorphism snows also that $D$ is a homomorphism, except that $O^{(2)}(4,2)$ must be treated separately. See Dieudonné [5].)

THEOREM 4.4. The Wall norm $N_{w}: U_{\varphi}(E, q) \rightarrow \Delta / \Sigma \Psi\left[\Delta, \Omega_{w}\right]$ is a homomorphism.

Proof. (A) When $k$ is GF(2) the factor group is trivial, so we may assume that $U(E, q)$ is not $O^{(2)}(4,2)$ and that $U_{\varphi}(E, q)$ is generated by transvections when $k$ is a field and by quasi-symetries when $k$ is not a field (Lemma 2.12). So it will suffice to prove the following:

"Suppose $X$ is a finite dimensional isometry of $(E, q)$ and $P$ is a transvection or quasi-symmetry of $(E, q)$ according as $k$ is or is not a field. Then

$$
N_{w}(P X)=N_{w}(P) N_{w}(X) " .
$$


Put $P=1+a \alpha^{-1} a$, where $a \in E^{\#}$ is isotropic or non-isotropic according as $k$ is or is not a field, and $f(a, w)=0$ or 1 , and $\alpha \in q(a)$.

Then $N_{w}(P)=\alpha^{-1} \Sigma \Psi\left[\Delta, \Omega_{w}\right]$. Let $(V, g)$ be the Cayley space and form of $X$.

(B) Suppose $a \neq V+R(E)$. Choose $\left(a_{1}, \ldots, a_{p}\right)$, any basis of $V$ (when $k$ is a field) or any w-basis of $v$ upper triangularizing $g$ (when $k$ is not a field) and put

$$
[g]=A \text { or }[g]=\left[\begin{array}{llll}
\alpha_{1} & & & \\
& \ddots & * \\
& \ddots & \\
& 0 & \ddots & \\
& & & \alpha_{r}
\end{array}\right] \text {. }
$$

So $N_{w}(X)=\operatorname{det} A$ or $N_{w}(X)=\alpha_{1}^{-1} \ldots \alpha_{p}^{-1}$, modulo $\Sigma \Psi\left[\Delta, \Omega_{w}\right]$. By Lemma 2.11, the Cayley form of $P X$ has the form

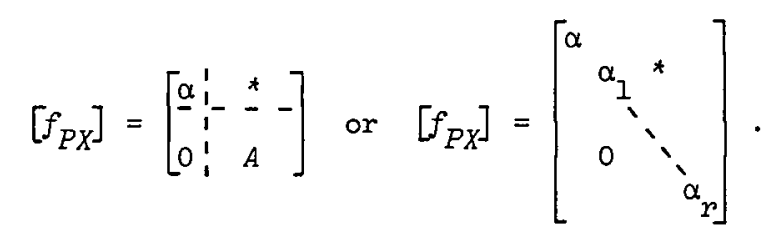

So $N_{w}(P X)=\alpha(\operatorname{det} A)$ or $N_{w}(P X)=\alpha^{-1} \alpha_{1}^{-1} \ldots \alpha_{r}^{-1}$, modulo $\Sigma \Psi\left[\Delta, \Omega_{w}\right]$. So $N_{w}(P X)=N_{w}(P) N_{w}(X)$.

(C) Suppose $a \in V+R(E)$. Put $a=b+t$ where $b \in V^{\#}$ and $t \in R(E)$ and suppose first that $b$ is not $g$-isotropic. Then we can complete $b$ to a $w$-basis $\left(b, a_{2}, \ldots, a_{p}\right)$ of $V$ so that $g$ has the form

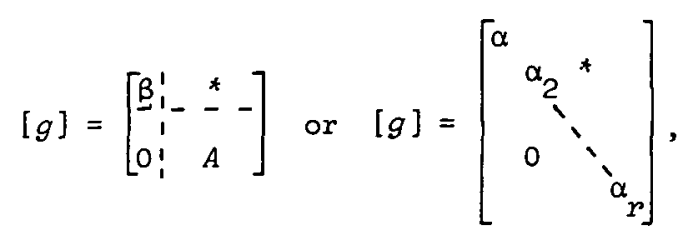

where $(\alpha+\beta)+l \in m$. So $N_{w}(X)=\beta(\operatorname{det} A)$ or $\beta^{-1} \alpha_{2}^{-1} \ldots \alpha_{r}^{-1}$ modulo 
$\Sigma \Psi\left[\Delta, \Omega_{\omega}\right]$. Let $Y$ be the isometry with space $\left(a_{2}, \ldots, a_{r}\right\rangle$ and form the corresponding restriction of $g$; then $X=\left(1+b \beta^{-1} b\right) Y$.

When $\beta=\alpha^{J}$ then $t=0$ and $P X=Y$, also $\alpha^{-1} B^{-1} \in \Psi$ and so $N_{w}(P X)=N_{w}(P) N_{w}(X)$. When $B \neq \alpha^{J}$ then

$$
P X=\left\{1+(\alpha+\lambda t) \alpha^{-1}(\alpha+\beta+f(a, a)) \beta^{-1}(\alpha+\lambda t)\right\} y,
$$

where $\lambda=\beta(\alpha+\beta+f(a, a))^{-1}(\alpha+f(a, a)) \beta^{-1}$. Now $\alpha^{-1}(\alpha+\beta+f(a, a)) \beta^{-1} \in \alpha^{-1} B^{-1} \Sigma$ and again $N_{w}(P X)=N_{w}(P) N_{w}(X)$.

(D) Suppose now that $g(b, b)=0$ so that $b$ is singular. Hence $\alpha$ is isotropic and by assumption $k$ is a field, also $\alpha \in m^{\#}$ and so $N_{w}(P)=1$. Choose $c \in V$ so that $g(c, b)=1$ and $g(b, c) \neq 0$, and complete $(b, c)$ to a basis $\left(b, c, a_{3}, \ldots, a_{p}\right)$ of $v$ so that $g$ has the form

$$
[g]=\left[\begin{array}{cc:c}
0 & \beta & * \\
\underline{I} & -\gamma_{1} & - \\
\hdashline 0 & A
\end{array}\right],
$$

where $B=1+f(b, c)$ and $\gamma=q(c)$. So $N_{w}(X)=B(\operatorname{det} A)$ modulo $\Sigma \Psi\left[\Delta, \Omega_{w}\right]$. Let $Y$ be the isometry with space $\left(a_{3}, \ldots, a_{p}\right)$ and form the corresponding restriction of $g$; then $X=\left(1+b \gamma \beta^{-1} b+b c+c \beta^{-1} b\right) Y$ and so

$$
P X=\left(1+b\left(\gamma \beta^{-1}+\alpha^{-1} \beta^{-1}\right) b+b\left(c+\alpha^{-1} t\right)+\left(c+\alpha^{-1} t\right) \beta^{-1} b\right) y .
$$

Hence the matrix of the form of $P X$ with respect to the basis $\left(b, c+\alpha^{-1} t, a_{3}, \ldots, a_{r}\right)$ is

$$
\left[f_{P X}\right]=\left[\begin{array}{cc:c}
0 & \beta & * \\
1 & \gamma \pm \alpha_{-1}^{-1} & - \\
\hdashline 0 & A
\end{array}\right]
$$

and so $N_{w}(P X)=N_{w}(X)$ as required. 
THEOREM 4.5. Suppose $q$ is indefinite and $e \in E^{\#}$ is singular. If $k$ is not a fiezd or if $q$ is defective, then the Wall norm $N_{e}$ is an epimorphism with kernel $T(E, q)$ and so

$$
U_{\varphi}(E, q) / T(E, q)=\Delta / \Sigma[\Delta, \Omega] \text {. }
$$

If $k$ is a field and $q$ is non-defective then the restriction of the wall norm (spinor norm) to $S O(E, q)$ is an epimorphism onto $\Delta / \Psi$, and if $n \geq 3$ this restriction has kernel $T(E, q)$ and

$$
o_{\varphi}(E, q) / T(E, q)=(\Delta / \Psi) \times \mathrm{Z}_{2} .
$$

Proof. (A) The final isomorphism follows from the earlier statements since the factor group of $O_{\varphi}(E, q)$ modulo the intersection of $S O(E, q)$ with kernel of the spinor norm has exponent 2 .

Choose $(e, d)$ a hyperbolic pair in $(E, q)$; then for all $\lambda \in \Delta$ the isometry $1+\left(\lambda^{-1} e+d\right) \lambda\left(\lambda^{-1} e+d\right)$ has Wall norm $\lambda \Sigma \Psi[\Delta, \Omega]$ with respect to $e$. When $k$ is a field and $q$ is non-defective, the product of this isometry with $1+(e+d)(e+d)$ lies in $S O(E, q)$ and has spinor norm $\lambda \Psi$.

When $k$ is not a field then $T(E, q)$ is generated by semi-singular transvections, and it is clear that each of these has trivial Wall norm. When $k$ is a field every semi-singular transvection and quasi-transvection has trivial wall norm, and if also $q$ is non-defective, every quasitransvection lies in $S O(E, q)$, having dimension 2 .

It remains to show that the kernel of $N_{e}$, intersected with $S O(E, q)$ in the case when $k$ is a field and $q$ is non-defective, lies in $T(E, q)$.

(B) For all $\lambda \in k^{\#}, \lambda \neq 1$, we calculate the Wall norm with respect to $e$ of the isometry $P$ with Cayley space $\langle e, d\rangle$ defined by

$$
\begin{gathered}
e P=\lambda e, \\
d P=\left(\lambda^{-1}\right)^{J} d,
\end{gathered}
$$

by using the w-basis $(a, b)$ of $(e, d)$ upper triangularizing the Cayley form of $P$, where 


$$
\begin{gathered}
a=\left(\lambda^{-1}+1\right) e+d, \\
b=(\lambda+1) e+d .
\end{gathered}
$$

Now $P=1+e\left(\left(\lambda^{-1}\right)^{J}+1\right) d+d(\lambda+1) e$ and so with respect to this new basis

$$
\left[f_{P}\right]=\left[\begin{array}{cc}
\left(\lambda^{-1}+1\right)(\lambda+1)^{-1}+1 & * \\
0 & 1+\left(\left(\lambda^{-1}\right)^{J}+1\right)^{-1}\left(\lambda^{J}+1\right)
\end{array}\right] .
$$

Hence $N_{e}(P)=\lambda \Sigma \Psi[\Delta, \Omega]$ (after calculation).

Suppose the rank of $f$ is 2 , so that $\Sigma \Psi[\Delta, \Omega]=\Sigma \Psi$. If $P$ lies in the kernel of $N_{e}$, and also in $S O(E, q)$ in the case when $k$ is a field and $q$ is non-defective, then we can choose $X \in T(E, q)$ by Lemma 3.7 so that $P X$ fixes both $\langle e\rangle$ and $\langle d\rangle$. By the above calculation and Lemma 3.4, it follows that $P X$ and hence $P$ lies in $T(E, q)$ as required.

(C) The proof when the rank of $f$ is greater than 2 can now be followed in Wall [10], 55 . We have already proven $(\alpha)$ in Lemma 3.7. The proof of $(\beta)$ given on page 210 will suffice when $a$ is non-isotropic; when $\alpha$ is isotropic then $\alpha$ is symmetric and so $\lambda^{-1} \alpha^{-1}\left(\lambda^{-1}\right)^{J} \in q\left(\lambda^{-1} \alpha^{-1} a\right)$. So by Lemma 3.4 we can choose $Q \in T(E, q)$ with Cayley space in $(e, d)$ so that

$$
\begin{gathered}
e Q=\lambda^{-1} \alpha^{-1}\left(\lambda^{-1}\right)^{J} \alpha e, \\
d Q=\lambda^{J} \alpha \lambda \alpha^{-1} d,
\end{gathered}
$$

and then by part (B),

$$
N_{e}^{*}(Q)=\lambda^{-1} \alpha^{-1}\left(\lambda^{-1}\right)^{J} \alpha \Sigma=[\lambda, \alpha] \Sigma .
$$

Finally in the proof of the theorem from $(\alpha)$ and $(\beta)$ on page 208, it will suffice to express $R$ as any product of one-dimensional isometries rather than as a semi-direct product (the exceptional case $O^{(2)}(4,2)$ is generated by transvections and singular 2-transvections, but the singular 2-transvections already lie in $T(E, q)$ ).

COROLLARY 4.6 (a) (cf. Dieudonne [2] and [3], 513). If the witt 
index of $q$ is at least 2 , then the factor group of $U_{\varphi}(E, q)$ modulo $T(E, q)$ is abelian.

(b) (af. Seip-Hornix [8], 9.5 and Dieudonné [2], §15). If $k$ is $a$ quatemionic division ring and $n=2$ and $q$ is indefinite, then the factor group of $U(E, q)$ modulo $T(E, q)$ is isomorphic to the factor group of $\Delta$ modulo the multiplicative group of $z$, and so is not abelian.

(c) (cf. Wall [10], Theorem 2, and Seip-Hornix [8], 11.4 and Remarks) If $k$ is a finite dimensional algebra over $z$ and $q$ is indefinite and $n \geq 3$, then the factor group of $U_{\varphi}(E, q)$ modulo $T(E, q)$ has exponent 2 and so is abelian.

Proof. We need only prove part (c) when $k$ is not a field - the argument is the same as Wall [10], part 2, and involves showing that $\Delta / \Sigma[\Delta, \Omega]$ has exponent 2 . Choose $a \in E$ non-singular orthogonal to some hyperbolic plane of $(E, q)$ and choose $\alpha \in q(a)$; then $\alpha \notin z$. Then the spaces $\alpha_{z}+\tau$ and $s$ have $z$-dimensions $1+z=(m-1)$ and $l_{2} m(m+1)$ respectively, and $\Omega$ contains every non-zero element of $\alpha z+l$.

Suppose $\mu \in \Delta$. Since the $z$-dimension of $k$ is only $m^{2}$, the spaces $\mu(\alpha z+l)$ and $s$ intersect non-trivially. Choose $\mu \lambda$ in this intersection; then $\mu \lambda=\lambda^{J} \mu^{J}$ and so

$$
\left(\lambda^{-1} \mu \lambda \mu^{-1}\right) \mu^{2}=\lambda^{-1} \lambda^{J} \mu^{J} \mu
$$

Also the spaces $\lambda s$ and $s$ both have $z$-dimension $z(m+1)$, so we can choose $\lambda \nu$ non-zero in the intersection. Then $\lambda \nu=v \lambda^{J}$ and so

$$
\lambda^{-1} \lambda^{J}=\lambda^{-1} \nu^{-1} \lambda \nu
$$

Since $\lambda \in \Omega$, we conclude that $\mu^{2} \in \Sigma[\Delta, \Omega]$ and so $\Sigma[\Delta, \Omega]$ has exponent 2 .

\section{References}

[1] Richard Braver, "On a theorem of H. Cartan", Buzl. Amer. Math. Soc. 55 (1949), 619-620. 
[2] Jean Dieudonné, "On the structure of unitary groups", Trans. Amer. Math. Soc. 72 (1952), 367-385.

[3] Jean Dieudonné, "On the structure of unitary groups (II)", Amer. J. Math. 75 (1953), 665-678.

[4] Jean Dieudonné, "Sur les générateurs des groupes classiques", Srmma Brasiz. Math. 3 (1955), 149-179.

[5] Jean Dieudonné, La géométrie des groupes classiques, 2 éd. (Springer-Verlag, Berlin, Göttingen, Heidelberg, 1963).

[6] B. Huppert, Endliche Gruppen I (Die Grundlehren der mathematischen Wissenschaften, Band 134. Springer-Verlag, Berlin, Heidelberg, New York, 1967).

[7] Kenkiti Iwasawa, "Über die Einfachheit der speziellen projektiven Gruppen", Proc. Imp. Acad. Tokyo 17 (1941), 57-59.

[8] E.A.M. Seip-Hornix, "Clifford algebres of quadratic quaternion forms. I", K. Nederl. Akad. Wetensch. Proc. Ser. A 68 (1965), 326-344; "Clifford algebras of quadratic quaternion forms. II", $K$. Nederl. Akad. Wetensch. Proc. Ser. A 68 (1965), 345-363.

[9] J. Tits, "Classification of algebraic semisimple groups", AZgebraic groups and discontinuous subgroups (Proc. Sympos. Pure Math. Boulder, Colo., 1965, 33-62. Amer. Math. Soc., Providence, Rhode Island, 1966).

[10] G.E. Wall, "The structure of a unitary factor group", PubZ. Math. Inst. Hautes Études Scient. 1 (1959).

[11] G.E. Wall, "On the conjugacy classes in the unitary, symplectic and orthogonal groups", J. Austral. Math. Soc. 3 (1963), 1-62.

Department of Pure Mathematics, University of Sydney, Sydney, New South Wales. 